\title{
Effects of tanshinone IIA on the regulation of renal proximal tubular fibrosis
}

\author{
LUOYUAN CAO, BAOYING HUANG, XIANGUO FU, JING YANG, YINGHUA LIN and FENG LIN \\ Department of Central Laboratory, Ningde Municipal Hospital, Affiliated Hospital \\ of Fujian Medical University, Ningde, Fujian 352100, P.R. China
}

Received March 2, 2016; Accepted February 21, 2017

DOI: $10.3892 / \mathrm{mmr} .2017 .6498$

\begin{abstract}
The development of diabetes mellitus, along with its complications, is a chronic inflammatory response process. Chronic kidney diseases are characterized by renal fibrosis, and fibrosis is an important pathway for end-stage renal failure. According to previous studies, high glucose (HG) has been demonstrated to be the most important fibrogenesis-inducing agent. Tanshinone IIA is one of the main components isolated from Danshen (Salvia miltiorrhiza). Although tanshinone IIA has been widely used for the treatment of cardiovascular diseases, the possible role of tanshinone IIA in fibrosis regulation remains to be elucidated and requires investigation. In the present study, renal proximal tubular epithelial cells (HK-2) were treated with HG (30 mM glucose) to determine whether tanshinone IIA (1, 10 and $50 \mu \mathrm{M}$ ) had an effect on the regulation of renal cellular fibrosis. The results demonstrated that $50 \mu \mathrm{M}$ tanshinone IIA may exert optimal inhibitory effects on HG-induced Snail, fibronectin, vimentin and $\alpha$-smooth muscle actin ( $\alpha$-SMA) expression in HK-2 cells after 48 h. Tanshinone IIA also reversed $\mathrm{HG}$-induced morphological alterations in $\mathrm{HK}-2$ cells and inhibited an $\mathrm{HG}$-induced increase in fibronectin and $\alpha$-SMA mRNA and protein and an HG-induced decrease in E-cadherin. Furthermore, tanshinone IIA suppressed an HG-induced increase in Snail, which is a transcription factor that can suppress E-cadherin expression. E-cadherin is a major component of adherens junctions and a characteristic of epithelial integrity. Tanshinone IIA reversed HG-induced increase in $\alpha$-SMA and decrease in E-cadherin. These data suggest that tanshinone IIA has the potential to inhibit HG-induced renal tubular epithelial cell fibrosis possibly through the epithelial-myofibroblast transdifferentiation pathway. Therefore, tanshinone IIA may be considered a renoprotective agent for the treatment of renal fibrosis.
\end{abstract}

Correspondence to: Professor Baoying Huang, Department of Central Laboratory, Ningde Municipal Hospital, Affiliated Hospital of Fujian Medical University, 7 Jiaocheng Road, Ningde, Fujian 352100, P.R. China

E-mail: 18259375782@126.com

Key words: tanshinone IIA, renal fibrosis, epithelial-mesenchymal transition, HK-2, high glucose

\section{Introduction}

The development of diabetes mellitus (DM) is a chronic inflammatory response process, and chemokines serve an important role in the course of events (1). Hyperglycemia is a major factor leading to diabetic nephropathy (DN), which may lead to renal fibrosis. Drugs are usually administered to regulate blood glucose levels and to help prevent the progression of renal disease; however, drugs are not able to completely delay the development of DN. Consequently, novel therapeutic agents for the treatment and prevention of DN progression are required. During the epithelial-mesenchymal transition (EMT) process, cellular polarity is removed from the epithelial cells and the transdifferentiation of epithelial cells into mesenchymal cells occurs. E-cadherin and cytokeratin are markers of epithelial cells, whereas a-SMA and vimentin are markers of mesenchymal cells (2). Kalluri and Neilson (3) demonstrated that during the course of kidney fibrosis in mice, $\sim 12 \%$ of fibroblasts are derived from the bone marrow and $\sim 30 \%$ are derived due to EMT of tubular epithelial cells in the kidney. E-cadherin and $\alpha$-SMA are the most important proteins involved in EMT (4).

During the EMT process, a significant decrease in E-cadherin and an increase in $\alpha$-SMA expression has long been demonstrated, along with an increase in Snail protein (5). Certain transdifferentiating cells express $\alpha$-SMA, which is completely absent in tubular epithelial cells. The appearance of $\alpha$-SMA in transdifferentiating cells during EMT is a characteristic of myofibroblast formation (6). The loss of E-cadherin expression together with the simultaneous upregulation of vimentin expression is known to be a marker of EMT changes in epithelial cells (7). Vimentin is a type III intermediate filament protein that is normally found in mesenchymal cells, although it is occasionally expressed in migratory epithelial cells during embryogenesis and wound healing (8).

Tanshinone IIA $\left(\mathrm{C}_{19} \mathrm{H}_{18} \mathrm{O}_{3}\right)$ is a fat-soluble, pharmacologically active component of the herb Salvia miltiorrhiza, which has long been used for the treatment of renal disease in China. Previous studies (9-12) have demonstrated that tanshinone IIA inhibits the proliferation and migration of arterial smooth muscle cells, reduces pulmonary artery pressure, ameliorates hypoxia-induced pulmonary artery remodeling and attenuates interleukin-17A-induced systemic sclerosis in patient-derived dermal vascular smooth muscle cell activation (13). In 
addition, it has been demonstrated that tanshinone IIA effectively inhibits the proliferation and phenotypic transformation of rat cardiac fibroblasts, delays the progression of myocardial fibrosis and attenuates bleomycin-induced pulmonary fibrosis in rats $(14,15)$.

The present study used an in vitro model to elucidate the effects of tanshinone IIA on renal fibrosis and identified that tanshinone IIA serves an important role in the regulation of renal tubular epithelial cell fibrosis. Tanshinone IIA has also been shown to inhibit high glucose (HG)-induced renal tubular epithelial cell fibrosis possibly through the EMT pathway.

\section{Materials and methods}

Cell culture. The HK-2 cell line (CRL-2190; American Type Culture Collection, Manassas, VA, USA) consists of proximal tubular cells derived from a normal human kidney. The cells $\left(5 \times 10^{5}\right)$ were seeded in $25 \mathrm{~T}$ flasks (Corning Incorporated, Corning, NY, USA) and cultured with Dulbecco's modified Eagle's medium (DMEM) nutrient mixture (Gibco; Thermo Fisher Scientific, Inc., Waltham, MA, USA) supplemented with $10 \%$ (v/v) fetal bovine serum (FBS; Gibco; Thermo Fisher Scientific, Inc.), 2\% (v/v) penicillin/streptomycin (HyClone; GE Healthcare Life Sciences, Logan, UT, USA) and 1\% (v/v) L-glutamine at $37^{\circ} \mathrm{C}$ in an atmosphere containing $95 \%$ environmental air and $5 \% \mathrm{CO}_{2}$. The cells were trypsinized using $0.05 \%$ trypsin-EDTA (Gibco; Thermo Fisher Scientific, Inc.). Prior to exposure to high glucose, cells were cultured in a minimum starvation media $(0.1 \% \mathrm{FBS})$ for $24 \mathrm{~h}$. And then the cells were cultured in DMEM containing $5.5 \mathrm{mM}$ D-glucose (normal glucose, NG) as control group, $30 \mathrm{mM}$ mannitol (Harbin Pharmaceutical Group, Harbin, China) or $30 \mathrm{mM}$ D-glucose (Gibco; Thermo Fisher Scientific, Inc.) in culture flasks for $48 \mathrm{~h}$ to induce cellular fibrogenesis, and were subsequently treated with various concentrations of tanshinone IIA (98.0\% pure; Xi'an Hongsheng Biotech Company, Xi'an, China) over $48 \mathrm{~h}$. Three wells were allocated for each treatment, including a negative control (untreated cells). Tanshinone IIA was prepared as a 1-mM stock solution in dimethyl sulfoxide and stored in the dark at $-20^{\circ} \mathrm{C}$.

Cell viability assay. To detect apoptosis in vitro, TUNEL assays were performed using a one-step TUNEL fluorescent kit (Beyotime Institute of Biotechnology, Haimen, China). HK-2 cells were permeabilized with $0.1 \%$ Triton X-100, followed by fluorescein isothiocyanate-labeled TUNEL staining for $1 \mathrm{~h}$ at $37^{\circ} \mathrm{C}$. TUNEL-positive cells were imaged under a fluorescent microscope and quantified as the number of green spots in each image (x100 magnification); three images were counted using Cellsens Imaging software version 1.4.1 (Olympus Corporation, Tokyo, Japan) in each group. In addition, treated cells were lysed using a lysis buffer $[10 \mathrm{mM}$ Tris, $1 \mathrm{mM}$ EDTA, 1\% Triton X-100, $1 \mathrm{mM} \mathrm{Na}_{3} \mathrm{VO}_{4}, 20 \mu \mathrm{g} / \mathrm{ml}$ aprotinin, $20 \mu \mathrm{g} / \mathrm{ml}$ leupeptin, $1 \mathrm{mM}$ dithiothreitol and $50 \mathrm{mM}$ phenylmethane sulfonyl fluoride (PMSF)] and a bicinchoninic acid protein assay kit (cat. no. P0010S; Beyotime Institute of Biotechnology) was used to detect protein content.

RNA extraction and reverse transcription-quantitative polymerase chain reaction ( $R T-q P C R)$. RNAiso Plus reagent
(Takara Biotechnology Co., Ltd., Dalian, China) was used to isolate total RNA from the cultured cells in accordance with the manufacturer's protocol. RNA $(1 \mu \mathrm{g})$ from each sample was then reverse transcribed using an RNA PCR kit (Takara Biotechnology Co., Ltd.) according to the manufacturer's protocol. The resulting cDNAs were processed using RT-qPCR with SYBR-Green technology (Takara Biotechnology Co., Ltd.) according to manufacturer's protocol. Briefly, reactions were conducted in a StepOnePlus real-time PCR system (Applied Biosystems; Thermo Fisher Scientific, Inc.) for 40 cycles $\left(95^{\circ} \mathrm{C}\right.$ for $5 \mathrm{sec}$ and $60^{\circ} \mathrm{C}$ for $40 \mathrm{sec}$ ) following an initial $10 \mathrm{~min}$ incubation at $95^{\circ} \mathrm{C}$ in a $20 \mu \mathrm{l}$ volume. Subsequently, the quantification cycle $(\mathrm{Cq})$ was determined and the relative expression levels of Snail, fibronectin and E-cadherin were calculated based on the $\mathrm{Cq}$ values that were normalized to GAPDH in each sample. The relative gene expression levels were calculated using a comparative $\mathrm{Cq}$ method formula $2^{-\Delta \Delta \mathrm{Cq}}$ method (16). The primers for the RT-qPCR reaction were purchased from Sangon Biotech Co., Ltd. (Shanghai, China). The sequences were as follows: Snail, sense 5'-CATTCCACG CCCAGCTACCC-3', antisense 5'-CGCCCAGGCTCACAT ATTCC-3'; fibronectin, sense 5'-GTGATCTACGAGGGA CAGC-3', antisense 5'-GCTGGTGGTGAAGTCAAAG-3'; E-cadherin, sense 5'-GGGCTTGGATTTTGAGGC-3', antisense 5'-AGATGGGGGCTTCATTCAC-3'; GAPDH, sense 5'-ATGCTGGTGCTGAGTATGTC-3', antisense 5'-AGTTGT CATATTTCTCGTGG-3'; and $\alpha$-SMA sense 5'-AACTGTG AATGTCCTGTG-3' and antisense 5'-CATAGGTAACGA GTCAGAG-3'.

Western blot analysis. Western blot analysis was used to evaluate the protein expression levels of fibronectin, $\alpha$-SMA, vimentin, Snail and E-cadherin. The HK-2 cells were lysed using a lysis buffer $(10 \mathrm{mM}$ Tris, $1 \mathrm{mM}$ EDTA, $1 \%$ Triton $\mathrm{X}-100,1 \mathrm{mM} \mathrm{Na} \mathrm{VO}_{4}, 20 \mu \mathrm{g} / \mathrm{ml}$ aprotinin, $20 \mu \mathrm{g} / \mathrm{ml}$ leupeptin, $1 \mathrm{mM}$ dithiothreitol and $50 \mathrm{mM}$ PMSF). Bicinchoninic acid protein assay kit (cat. no. P0010S; Beyotime Institute of Biotechnology) was used to detect protein content. Equal quantities of protein $(30 \mu \mathrm{g})$ from each sample were separated by 8 or $10 \%$ SDS-PAGE, the protein was transferred onto a polyvinylidene difluoride (PVDF) membrane and the PVDF membrane was blocked with $10 \%$ (w/v) non-fat milk in Tris-buffered saline with $0.1 \%$ Tween-20 for $1 \mathrm{~h}$ at room temperature. The blots were probed overnight at $4^{\circ} \mathrm{C}$ with the following primary antibodies (all purchased from Abcam, Cambridge, UK) diluted to 1:2,000 (v/v): E-cadherin (ab133597), vimentin (ab184631), Snail (ab167609), fibronectin (ab194395) and $\alpha$-SMA (ab124964). Following hybridization, the blots were washed and hybridized with 1:6,000 (v/v) dilutions of a goat anti-rabbit immunoglobulin $\mathrm{G}(\mathrm{IgG})$ or goat anti-mouse IgG horseradish peroxidase-conjugated secondary antibody (cat. no. A0208 and A0216; Beyotime Institute of Biotechnology) at room temperature for $1 \mathrm{~h}$. Immunodetection was performed using enhanced chemiluminescence reagents (Beyotime Institute of Biotechnology). The blots were scanned and the intensity of each band was quantified using Image J software version 1.48u (National Institutes of Health, Bethesda, MD, USA). Signals were normalized against $\beta$-actin or GAPDH diluted to 1:1,500 (v/v) (cat. no. sc-130301 and sc-47724; Santa Cruz Biotechnology, Inc., Dallas, TX, USA) 
and the results were expressed as a relative expression of the control signal.

Immunocytochemistry. Following different treatments, HK-2 cells were plated onto 6-well plates with polylysine-coated coverslips at a density of $1.6 \times 10^{5}$ cells/well. Cells were fixed in $4 \%$ paraformaldehyde for $15 \mathrm{~min}$ at $4^{\circ} \mathrm{C}$ and stained with anti-fibronectin antibodies diluted to 1:50 (v/v). Briefly, HK-2 cells were fixed, blocked with $10 \%$ skimmed milk, washed with phosphate-buffered saline (PBS), inactivate endogenous peroxidase with $0.3 \% \mathrm{H}_{2} \mathrm{O}_{2}$ for $15 \mathrm{~min}$ and then incubated for $1 \mathrm{~h}$ with anti-fibronectin, washed with PBS, incubated for $1 \mathrm{~h}$ with secondary antibody 1:50 (v/v) (cat. no. A0216; Beyotime Institute of Biotechnology), Dako Real $^{\mathrm{TM}}$ Envision $^{\mathrm{TM}}$ detection $^{\mathrm{T}}$ system (cat. no. P0203; Beyotime Institute of Biotechnology) was used for detection. All procedures were performed at room temperature.

Statistical analysis. All experiments were repeated at least three times. Data are presented as the mean \pm standard deviation. Data were analyzed using SPSS software version 13.0 (SPSS, Inc., Chicago, IL, USA). Statistical significance was assessed using analysis of variance followed by the least significant difference test for multiple comparisons and $\mathrm{P}<0.05$ was considered to indicate a statistically significant difference.

\section{Results}

Dose-dependent effects of tanshinone IIA and HG on cell survival rates and protein content in $H K-2$. The underlying effects of tanshinone IIA and HG on glucose-induced pharmacology were evaluated. Cell survival rates and protein content were analyzed in cells treated with glucose $(30 \mathrm{mM})$ and tanshinone IIA $(1,10$ and $50 \mu \mathrm{M})$. These observations demonstrated that treatment with tanshinone IIA or HG did not statistically affect cellular survival or protein content (Fig. 1).

$H G$-induced EMT in HK-2 cells. To investigate whether HG could induce EMT in HK-2 cells, cells were treated with DMEM containing $5.5 \mathrm{mM}$ D-glucose, $30 \mathrm{mM}$ mannitol or HG (30 mM glucose). Cells treated with $5.5 \mathrm{mM}$ glucose exhibited a typical epithelial cuboidal shape with a characteristic confluent monolayer and cobblestone morphology; however, cells that were exposed to HG exhibited distinct morphological alterations and possessed an elongated, spindle shape (Fig. 2C). The expression of the epithelial phenotypic marker E-cadherin was significantly decreased in the $\mathrm{HG}$ group ( $\mathrm{P}<0.05$; Fig. 3A) and mesenchymal phenotypic markers, $\alpha-S M A$ and vimentin, were markedly increased $(\mathrm{P}<0.05$; Fig. 3B and C). In addition, the expression of fibronectin was enhanced following treatment with $\mathrm{HG}$, according to immunocytochemistry and western blot analysis (Fig. 3B and D). No significant differences were identified between the mannitol and NG groups. The downregulation of E-cadherin and the concomitant upregulation of $\alpha$-SMA, vimentin and fibronectin in the tubular epithelial cells strongly supported the case for $\mathrm{HG}$ as a potent stimulus of EMT in HK-2 cells.

Effects of tanshinone IIA on glucose-induced changes in HK-2 cell shape. To examine whether tanshinone IIA

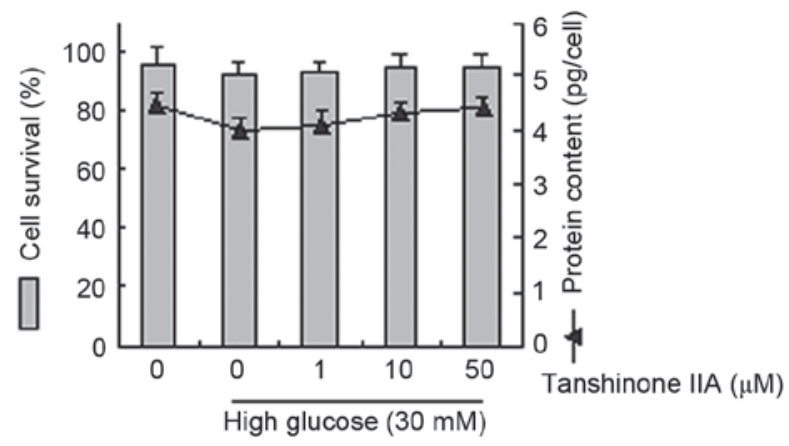

Figure 1. Effects of tanshinone IIA on HG-induced cell growth in HK-2 cells. Serum-deprived cells were treated with $\mathrm{HG}(30 \mathrm{mM})$ for $48 \mathrm{~h}$ in the presence of $10 \%$ fetal bovine serum with various concentrations of tanshinone IIA $(1,10$ and $50 \mu \mathrm{M})$ over $48 \mathrm{~h}$. It was evident that treatment with tanshinone IIA and HG did not significantly affect cell survival or protein content. HG, high glucose.

inhibits glucose-induced changes in HK-2 cells, cells were pretreated with glucose or mannitol for $48 \mathrm{~h}$, followed by tanshinone IIA at the indicated concentrations $(1,10$ and $50 \mu \mathrm{M}$ ) for $48 \mathrm{~h}$. To demonstrate the osmotic effects of glucose, mannitol was used as a control in the present study (Fig. 3). It is evident that $30 \mathrm{mM}$ glucose, but not mannitol, statistically increased the expression of protein markers. Therefore, it may be proposed that glucose (not osmosis) serves a pivotal role on the regulation of HK-2 cell fibrosis. HK-2 cells produced a confluent monolayer with cobblestone morphology in the control group (Fig. 2A and B). Following treatment with $30 \mathrm{mM}$ glucose, a marked number of cells exhibited an elongated, spindle shape (Fig. 2C, as indicated by arrows). As presented in Fig. 2D-F, cells were incubated with $30 \mathrm{mM}$ glucose for $48 \mathrm{~h}$ and treated with tanshinone IIA to investigate cellular morphology. It is evident that tanshinone IIA significantly reversed $\mathrm{HG}$-induced changes. In addition, tanshinone IIA appeared to be able to restore HK-2 cell epithelial morphology.

Tanshinone IIA suppresses HG-induced EMT in HK-2 epithelial cells. The expression of EMT marker proteins ( $\alpha$-SMA, vimentin and fibronectin) was examined. HG (30 mM) induced a notable increase in $\alpha$-SMA, vimentin and Snail expression, and a decrease in E-cadherin (Fig. 3). The aforementioned effects were significantly reversed by $50 \mu \mathrm{M}$ tanshinone IIA. Subsequently, $\alpha$-SMA, vimentin and Snail were decreased and E-cadherin was increased following treatment with tanshinone IIA in proximal tubule cells.

Effects of tanshinone IIA on Snail and E-cadherin synthesis in $\mathrm{HK}-2$ cells. In the HK-2 cells treated with HG (30 mM), the expression of Snail was significantly greater than that of cells treated with $5.5 \mathrm{mM}$ glucose or $30 \mathrm{mM}$ mannitol; however, the expression of Snail following pretreatment with tanshinone IIA was significantly decreased, as compared with HG treatment (Fig. 3C). It is evident that $30 \mathrm{mM}$ glucose, but not mannitol, significantly increased the expression of Snail. In addition, the mRNA expression levels of Snail were increased in HK-2 cells following treatment with $\mathrm{HG}$; however, the expression of Snail mRNA was reduced following treatment with $50 \mu \mathrm{M}$ 


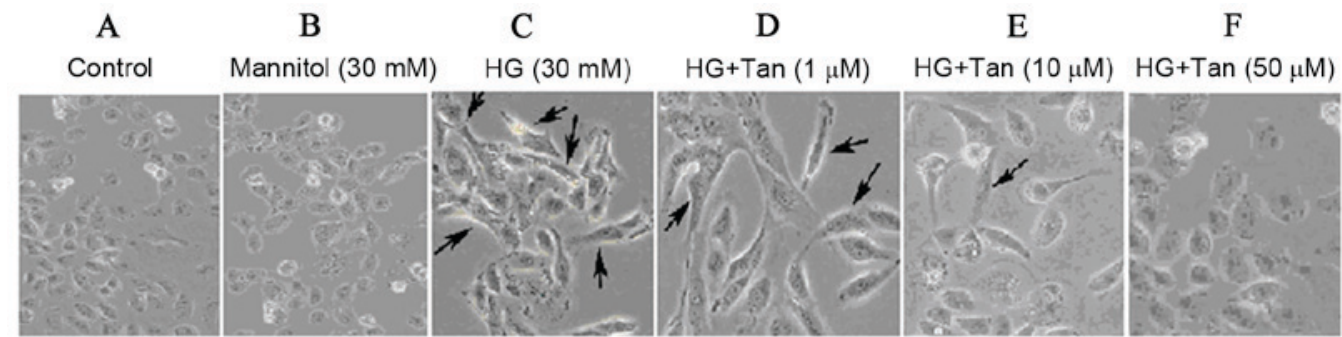

Figure 2. Tanshinone IIA-reversed HG-induced morphological alterations in HK-2 cells. HK-2 cells were incubated with HG (30 mM) for 48 h, and with various concentrations of tanshinone IIA $(1,10$ and $50 \mu \mathrm{M}$ ) for $48 \mathrm{~h}$. (A) Control HK-2 cells (treated with normal glucose, $5.5 \mathrm{mM}$ ) produced a confluent monolayer with cobblestone morphology. (B) Control group cells were treated with $30 \mathrm{mM}$ mannitol to determine the effects of osmosis. (C) Treatment with $30 \mathrm{mM} \mathrm{HG}$ was performed to induce epithelial-mesenchymal transition; a significant number of cells exhibited an elongated, spindle-shaped, fibroblastic morphology indicated by arrows. (D-F) Cells incubated with HG $(30 \mathrm{mM})$ and tanshinone IIA $(1,10$ and $50 \mu \mathrm{M})$ for $48 \mathrm{~h}$ exhibited morphological alterations. Tanshinone IIA treatment inhibited HG-induced morphologic changes and restored epithelial morphology in a dose-dependent manner (x200 magnification). HG, high glucose; Tan, Tanshinone IIA.

A
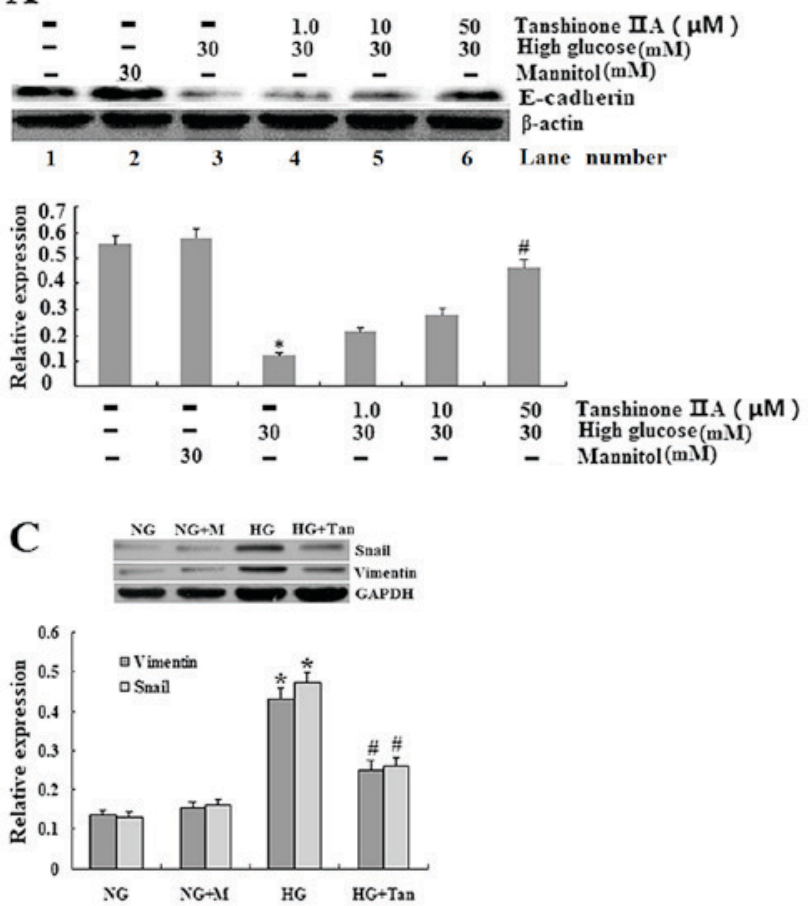

B

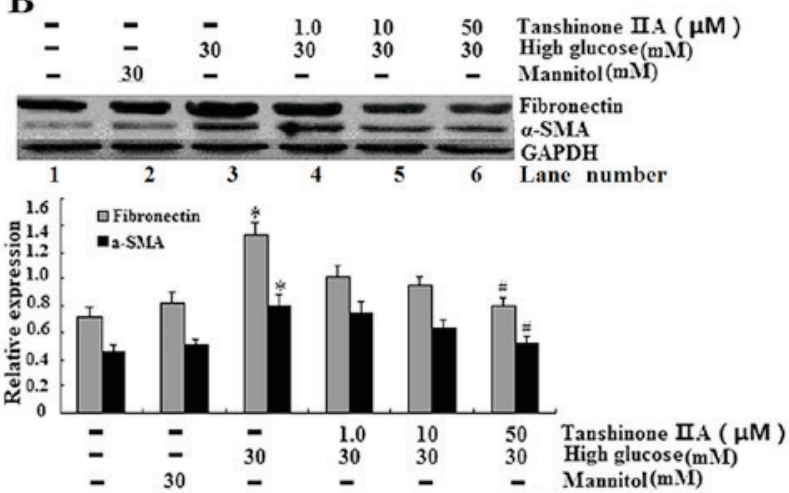

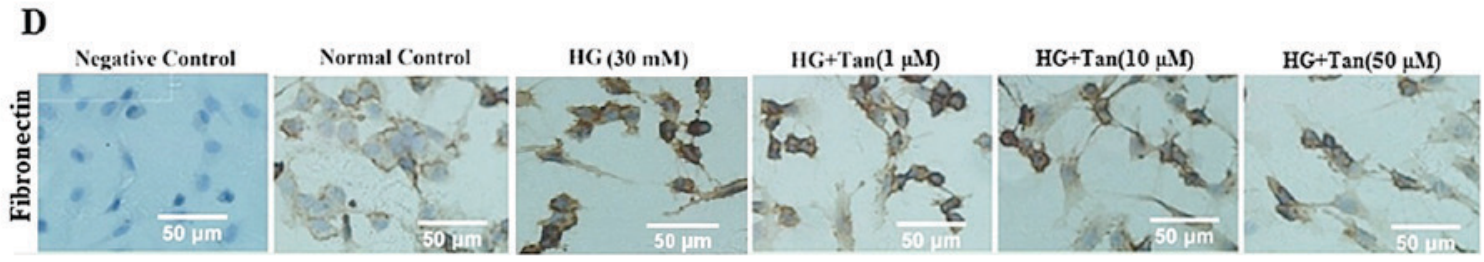

Figure 3. Expression of E-cadherin, $\alpha$-SMA, fibronectin, vimentin and Snail in HK-2 cells. (A and B) E-cadherin, $\alpha$-SMA and fibronectin expression was analyzed using western blotting for HK-2 cells cultured in $5.5 \mathrm{mM}$ glucose (lane 1), $30 \mathrm{mM}$ mannitol (lane 2), $30 \mathrm{mM}$ glucose (lane 3), $30 \mathrm{mM}$ glucose $+1 \mu \mathrm{M}$ tanshinone IIA (lane 4), $30 \mathrm{mM}$ glucose $+10 \mu \mathrm{M}$ tanshinone IIA (lane 5) and $30 \mathrm{mM}$ glucose $+50 \mu \mathrm{M}$ tanshinone IIA (lane 6). ${ }^{*} \mathrm{P}<0.05 \mathrm{vs}$. $5.5 \mathrm{mM}$ glucose-treated cells; ${ }^{~} \mathrm{P}<0.05$ vs. $30 \mathrm{mM}$ glucose-treated cells. (C) Vimentin and Snail expression was analyzed using western blotting for HK-2 cells cultured in $5.5 \mathrm{mM}$ glucose (NG group), $30 \mathrm{mM}$ mannitol (NG+M group), $30 \mathrm{mM}$ glucose ( $\mathrm{HG}$ group) and $30 \mathrm{mM}$ glucose $+50 \mu \mathrm{M}$ tanshinone IIA (HG+Tan group). "P $<0.05$ vs. NG group; ${ }^{\prime P}<0.05$ vs. HG group. (D) Expression of fibronectin in HK-2 cells, as determined using immunocytochemistry; HK-2 cells were starved (without FBS) and treated with glucose $(30 \mathrm{mM})$ for $48 \mathrm{~h}$ with tanshinone IIA $(1,10$ and $50 \mu \mathrm{M})$ for $48 \mathrm{~h}$. Values are presented as the mean \pm standard deviation. $\alpha$-SMA, $\alpha$-smooth muscle actin; $\mathrm{HG}$, high glucose; $\mathrm{NG}$, normal glucose.

tanshinone IIA (Fig. 4), which concomitantly increased E-cadherin protein expression, as the protein expression of E-cadherin is increasing in $\mathrm{HG}+\mathrm{Tan}(50 \mu \mathrm{M})$ group (Fig. 3A).
Effects of tanshinone IIA on EMT in HK-2 cells. EMT marker proteins (including $\alpha$-SMA, vimentin and fibronectin), epithelial phenotypic markers (E-cadherin) and transcriptional factors 
for EMT (Snail) were assessed using immunocytochemistry, western blotting and RT-qPCR. HG (30 mM) significantly increased extracellular fibronectin, $\alpha$-SMA and vimentin in HK-2 cells, compared with in the control group (Fig. 3; $\mathrm{P}<0.05)$. Notably, tanshinone IIA dose dependently (1, 10 and $50 \mu \mathrm{M})$ and significantly suppressed HG-induced expression of fibronectin, $\alpha$-SMA, vimentin and Snail. Detection of mRNA expression was performed using RT-qPCR. E-cadherin, fibronectin, $\alpha$-SMA and Snail mRNA expression was assessed in the control, $\mathrm{HG}$ and $\mathrm{HG}+$ tanshinone IIA groups (Fig. 4). E-cadherin mRNA expression was markedly reduced in the HG group, whereas Snail, fibronectin and $\alpha$-SMA mRNA expression was significantly increased. However, following treatment with $50 \mu \mathrm{M}$ tanshinone IIA, the mRNA expression levels were reversed, resulting in levels similar to those in the control group. HG increased the expression of EMT marker proteins, whereas tanshinone IIA significantly reversed the increase of EMT marker proteins in HK-2 cells. The aforementioned observation indicated that tanshinone IIA has the potential to downregulate HG-induced EMT in HK-2 cells.

\section{Discussion}

DN is a serious microvascular complication associated with DM. The degree of renal interstitial fibrosis is more closely associated with the progressive loss of renal functions, compared with diabetic glomerulopathy (1). Urinary glucose, proteinuria and cytokines stimulate renal tubular epithelial cells in patients with DN, causing the cells to be prone to structural and functional damage, and phenotypic modulation, which are considered to be the initiating factors for renal fibrosis. As for renal tubular epithelial cells, EMT is an important mechanism by which renal interstitial fibrosis occurs (5). EMT describes a process by which cells lose their relatively differentiated epithelial characteristics and demonstrate increased migratory or synthetic properties. This transition is evidenced by the loss of proteins involved in cell-cell junctions and an increase in proteins, including vimentin, fibronectin and $\alpha$-SMA (17). In the EMT process, a significant decrease in E-cadherin expression and an increase in $\alpha$-SMA expression have been demonstrated, along with an increase in Snail protein $(18,19)$. The present study demonstrated that HG significantly increased extracellular fibronectin, $\alpha$-SMA and vimentin expression in HK-2 cells, compared with in the control group (Fig. 3; $\mathrm{P}<0.05)$. Changes in E-cadherin were rapidly accompanied by an upregulation of mesenchymal markers. The loss of E-cadherin expression is the most common biochemical alteration associated with EMT. These results indicated that HG induced EMT in HK-2 cells.

In previous studies, tanshinone IIA has been reported to modulate growth, anti-inflammatory effects, proliferation and migration of numerous cells, including keratinocytes, RAW 264.7 cells, cardiac fibroblasts, cardiomyocytes, tumor cells and human aortic smooth muscle cells (10-12,20-23). The present study identified that the expression levels of fibronectin, $\alpha$-SMA, vimentin and Snail were markedly suppressed following treatment of HG-stimulated HK-2 cells with $50 \mu \mathrm{M}$ tanshinone IIA. Therefore, tanshinone IIA may be considered an effective treatment for HK-2 fibrosis.

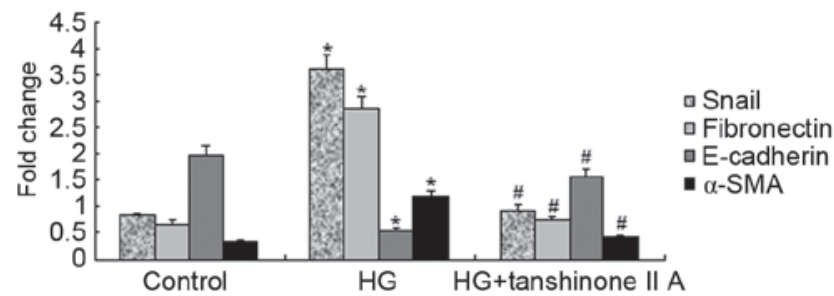

Figure 4. Tanshinone IIA inhibits HG-induced epithelial-mesenchymal transition-related mRNA expression in HK-2 cells, as determined using reverse transcription-quantitative polymerase chain reaction. The HK-2 cells were cultured in 25T flasks. Cells were starved (without FBS) and divided into three groups: Control group (5.5 mM glucose), $\mathrm{HG}$ group (30 $\mathrm{mM}$ glucose) and $\mathrm{HG}+$ tanshinone IIA $(50 \mu \mathrm{M})$ group. The $\mathrm{HG}+$ tanshinone IIA group was treated with $\mathrm{HG}$ for $48 \mathrm{~h}$, followed by treatment with $50 \mu \mathrm{M}$ tanshinone IIA. ${ }^{*} \mathrm{P}<0.05$ vs. control group; ${ }^{\prime} \mathrm{P}<0.05$ vs. $\mathrm{HG}$ group. $\alpha$-SMA, $\alpha$-smooth muscle actin; HG, high glucose.

It has previously been demonstrated that stimulating HK-2 with HG resulted in increased expression of fibronectin (24). Elevated glucose levels have been detected in renal fibrosis animal models and in patients with DN (25). Glucose also induced accumulation of extracellular matrix components, including fibronectin, in mesangial cells (26). These findings suggested that fibronectin may serve an important role in renal fibrosis.

In the present study, in HK-2 cells, the expression of fibronectin, $\alpha$-SMA and vimentin was significantly increased following treatment with $\mathrm{HG}(30 \mathrm{mM})$. Tanshinone IIA dose dependently $(1,10$ and $50 \mu \mathrm{M})$ and significantly, suppressed $\mathrm{HG}$-induced increases in the secretion levels of fibronectin, $\alpha$-SMA and vimentin (Fig. 3). The present study demonstrated that tanshinone IIA serves a pivotal role in the regulation of renal tubular fibrosis. Therefore, tanshinone IIA may be considered an effective therapeutic supplement for the treatment of renal tubular fibrosis by inhibiting fibronectin, $\alpha$-SMA and vimentin expression.

Snail is a zinc finger transcription factor that functions as a regulator to suppress the expression of adhesion molecules during EMT (27). The most common biochemical change associated with EMT is the loss of E-cadherin expression. According to Medici et al (28), Snail suppresses E-cadherin. In the present study, Snail expression was promoted in HK-2 cells under HG conditions; however, Snail expression was reversed by treatment with tanshinone IIA, which concomitantly increased E-cadherin protein. These results indicated that tanshinone IIA completely reversed $\mathrm{HG}$-induced increase in $\alpha$-SMA and decrease in E-cadherin. Therefore, tanshinone IIA may be considered an effective therapeutic compound for the treatment of proximal tubular fibrosis, since it regulates Snail expression and the EMT process.

In renal tubular epithelial cells, hyperglycemia-induced EMT may contribute to renal fibrosis. The present study used an in vitro model to elucidate the effects of tanshinone IIA on renal fibrosis. The results demonstrated that $\mathrm{HG}$ induced the EMT process in HK-2 cells; and notably, tanshinone IIA suppressed HG-induced EMT in HK-2 cells. The present study demonstrated that tanshinone IIA may decrease HG-induced HK-2 fibrosis by downregulating the expression of Snail to promote E-cadherin expression in HK-2 cells. These findings 
suggested that tanshinone IIA may be an effective therapeutic supplement for the treatment of renal tubular fibrosis via its ability to inhibit the EMT process.

In conclusion, the results of the present study demonstrated that tanshinone IIA serves a protective role against HG-induced renal tubular epithelial cell fibrosis. Tanshinone IIA antagonized HG-induced EMT signals, possibly by downregulating the expression of Snail in renal cells. Tanshinone IIA also increased the expression of E-cadherin and decreased the expression of $\alpha$-SMA, fibronectin and vimentin. Therefore, tanshinone IIA may exert the ability to inhibit HG-induced renal tubular epithelial cell fibrosis possibly by regulating the EMT pathway. Tanshinone IIA may be considered a renoprotective agent for the treatment of renal fibrosis in DN.

\section{Acknowledgements}

The present study was supported by research grants from the Fujian Science and Technology Project of Nature Science Foundation (grant no. 2012J01435), a special fund from the Fujian Medical University for Scientific and Technological Development (grant no. FZS13022Y) and the Youth Foundation from the Health Department of Fujian province (grant no. 2013-1-50).

\section{References}

1. Phillips AO: The role of renal proximal tubular cells in diabetic nephropathy. Curr Diab Rep 3: 491-496, 2003.

2. Câmara J and Jarai G: Epithelial-mesenchymal transition in primary human bronchial epithelial cells is Smad-dependent and enhanced by fibronectin and TNF-alpha. Fibrogenesis Tissue Repair 3: 2, 2010.

3. Kalluri R and Neilson EG: Epithelial-mesenchymal transition and its implications for fibrosis. J Clin Invest 112: 1776-1784, 2003.

4. Fragiadaki M and Mason RM: Epithelial-mesenchymal transition in renal fibrosis-evidence for and against. Int J Exp Pathol 92 : 143-150, 2011.

5. Carew RM, Wang B and Kantharidis P: The role of EMT in renal fibrosis. Cell Tissue Res 347: 103-116, 2012.

6. Sebe A, Leivonen SK, Fintha A, Masszi A, Rosivall L, Kähäri VM and Mucsi I: Transforming growth factor-betainduced alpha-smooth muscle cell actin expression in renal proximal tubular cells is regulated by p38beta mitogen-activated protein kinase, extracellular signal-regulated protein kinase 1,2 and the Smad signalling during epithelial-myofibroblast transdifferentiation. Nephrol Dial Transplant 23: 1537-1545, 2008.

7. Lee JM, Dedhar S, Kalluri R and Thompson EW: The epithelial-mesenchymal transition: New insights in signaling, development, and disease. J Cell Biol 172: 973-981, 2006.

8. Liu LK, Jiang XY, Zhou XX, Wang DM, Song XL and Jiang HB: Upregulation of vimentin and aberrant expression of E-cadherin/beta-catenin complex in oral squamous cell carcinomas: Correlation with the clinicopathological features and patient outcome. Mod Pathol 23: 213-224, 2010.

9. Wu WY, Yan H, Wang XB, Gui YZ, Gao F, Tang XL, Qin YL, Su M, Chen T and Wang YP: Sodium tanshinone IIA silate inhibits high glucose-induced vascular smooth muscle cell proliferation and migration through activation of AMP-activated protein kinase. PLoS one 9: e94957, 2014.

10. Zhang HH, Chen YC, Liang L and Zeng Z: Tanshinone IIA inhibits in vitro cellular proliferation and migration of vascular smooth muscle cell of rabbit. Sichuan Da Xue Xue Bao Yi Xue Ban 39: 188-192, 2008 (In Chinese).
11. Luo Y, Xu DQ, Dong HY, Zhang B, Liu Y, Niu W, Dong MQ and Li ZC: Tanshinone IIA inhibits hypoxia-induced pulmonary artery smooth muscle cell proliferation via Akt/Skp2/p27-associated pathway. PLoS one 8: e56774, 2013.

12. Li X, Du JR, Yu Y, Bai B and Zheng XY: Tanshinone IIA inhibits smooth muscle proliferation and intimal hyperplasia in the rat carotid balloon-injured model through inhibition of MAPK signaling pathway. J Ethnopharmacol 129: 273-279, 2010.

13. Liu M, Yang $\mathrm{J}$ and Li M: Tanshinone IIA attenuates interleukin-17A-induced systemic sclerosis patient-derived dermal vascular smooth muscle cell activation via inhibition of the extracellular signal-regulated kinase signaling pathway. Clinics (Sao Paulo) 70: 250-256, 2015.

14. Zhan CY, Tang JH, Zhou DX and Li ZH: Effects of tanshinone IIA on the transforming growth factor $\beta 1 /$ Smad signaling pathway in rat cardiac fibroblasts. Indian J Pharmacol 46: 633-638, 2014.

15. Zhou D, Li Z, Zhang L and Zhan C: Inhibitory effect of tanshinone IIA on TGF II- $\beta 1$-induced cardiac fibrosis. J Huazhong Univ Sci Technolog Med Sci 32: 829-833, 2012.

16. Livak KJ and Schmittgen TD: Analysis of relative gene expression data using real-time quantitative PCR and the 2(-Delta Delta C(T)) Method. Methods 25: 402-408, 2001.

17. Galichon P and Hertig A: Epithelial to mesenchymal transition as a biomarker in renal fibrosis: Are we ready for the bedside? Fibrogenesis Tissue Repair 4: 11, 2011.

18. Yu H, Shen Y, Hong J, Xia Q, Zhou F and Liu X: The contribution of TGF- $\beta$ in Epithelial-Mesenchymal Transition (EMT): Down-regulation of E-cadherin via snail. Neoplasma 62: 1-15, 2015.

19. He L, Lou W, Ji L, Liang W, Zhou M, Xu G, Zhao L, Huang C, Li R, Wang H, et al: Serum response factor accelerates the high glucose-induced Epithelial-to-Mesenchymal Transition (EMT) via snail signaling in human peritoneal mesothelial cells. PLoS one 9: e108593, 2014.

20. Fan GW, Gao XM, Wang H, Zhu Y, Zhang J, Hu LM, Su YF, Kang LY and Zhang BL: The anti-inflammatory activities of Tanshinone IIA, an active component of TCM, are mediated by estrogen receptor activation and inhibition of iNOS. J Steroid Biochem Mol Biol 113: 275-280, 2009.

21. Chen J, Shi DY, Liu SL and Zhong L: Tanshinone IIA induces growth inhibition and apoptosis in gastric cancer in vitro and in vivo. Oncol Rep 27: 523-528, 2012.

22. Li FL, Xu R, Zeng QC, Li X, Chen J, Wang YF, Fan B, Geng L and $\mathrm{Li} \mathrm{B}$ : Tanshinone IIA inhibits growth of keratinocytes through cell cycle arrest and apoptosis: Underlying treatment mechanism of psoriasis. Evid Based Complement Alternat Med 2012: 927658, 2012.

23. Chan P, Liu JC, Lin LJ, Chen PY, Cheng TH, Lin JG and Hong HJ: Tanshinone IIA inhibits angiotensin II-induced cell proliferation in rat cardiac fibroblasts. Am J Chin Med 39: 381-394, 2011.

24. Hsieh PF, Liu SF, Lee TC, Huang JS, Yin LT, Chang WT, Chuang LY, Guh JY, Hung MY and Yang YL: The role of IL-7 in renal proximal tubule epithelial cells fibrosis. Mol Immunol 50: 74-82, 2012.

25. Haneda M, Koya D, Isono M and Kikkawa R: Overview of glucose signaling in mesangial cells in diabetic nephropathy. J Am Soc Nephrol 14: 1374-1382, 2003.

26. Lan T, Liu W, Xie X, Huang K, Peng J, Huang J, Shen X, Liu P, Yang $\mathrm{H}$ and Huang $\mathrm{H}$ : Berberine suppresses high glucose-induced TGF- $\beta 1$ and fibronectin synthesis in mesangial cells through inhibition of sphingosine kinase 1/AP-1 pathway. Eur J Pharmacol 697: 165-172, 2012.

27. Zhu LF, Hu Y, Yang CC, Xu XH, Ning TY, Wang ZL, Ye JH and Liu LK: Snail overexpression induces an epithelial to mesenchymal transition and cancer stem cell-like properties in SCC9 cells. Lab Invest 92: 744-752, 2012.

28. Medici D, Hay ED and Olsen BR: Snail and Slug promote epithelial-mesenchymal transition through beta-catenin-T-cell factor-4-dependent expression of transforming growth factor-beta3. Mol Biol Cell 19: 4875-4887, 2008 